\title{
La microfinance : une solution à l'exclusion bancaire du secteur informel au Maroc
}

\section{EL GHMARI Imad ${ }^{1}$ et OUKASSI Mustapha ${ }^{2}$}

${ }^{1}$ Faculté des Sciences Juridiques, Economique et sociale - Souissi, Université Mohamed V. Rabat, Maroc Laboratoire : recherche en management des organisations, droit des affaires et développement durable

${ }^{2}$ Faculté des Sciences Juridiques, Economique et sociale - Souissi, Université Mohamed V. Rabat, Maroc Laboratoire : recherche en management des organisations, droit des affaires et développement durable

Résumé : Depuis sa création par Mohammed Yunus, la microfinance a fait couler beaucoup d'encre. Son implantation au Maroc a tout de suite été un succès puisqu'en quelques années, les Institutions de Microfinance (IMF) marocaines ont constitué un portefeuille extraordinaire. Cependant beaucoup d'IMF dans le monde, notamment au Maroc, arrivent à maturité et sont confrontées à des problématiques de financement et de développement de portefeuille de projet. Cet article étudie en profondeur la situation du microcrédit aujourd'hui au Maroc et son rôle dans le financement du secteur informel marocain.

Mots-clés : microfinance, microcrédit, secteur informel.

\section{Introduction}

Depuis plus de 30 ans, la notion de «secteur informel » domine le champ économique. Il investit autant dans les opérations que dans la science et les médias. La crise économique et le processus d'ouverture ont exacerbé l'importance du secteur informel, ce qui a ravivé la polémique. Bref, si l'on veut mieux comprendre le fonctionnement économique des pays en développement, il faut mieux comprendre le secteur informel. Cette connaissance reste un enjeu politique, économique et social (Cling et al., 2012). 
Nul ne peut nier l'importance du secteur informel dans le contexte de l'économie nationale. En fait, le marché informel a enregistré des performances quantitatives considérables et a fourni à un grand nombre de personnes des opportunités d'emploi, ce qui a nui au secteur formel légal et causé d'énormes pertes aux unités de production nationales. L'économie informelle au Maroc, en l'absence de données précises, est estimée entre 30 et $40 \%$ du revenu global et emploi ou fait vivre plus de la moitié de la population.

En fait, son analyse semble être un sujet très confus, posant un véritable défi aux économistes. Pendant longtemps, il y a eu des ambiguïtés dans l'origine et la dynamique du département, les termes et définitions proposés, et les fonctions qu'il remplit. Le secteur informel est un concept polysémique et expansible, il est soit assimilé à un secteur en voie d'assimilation, soit assimilé au sous-emploi et au chômage, aux activités marginales, soit encore assimilé à un petit secteur dominé et contrôlé par le secteur formel capitaliste. Activités de production. Au sens commun, elle est synonyme de pauvreté, d'activité de rue et d'absence de cadre réglementaire. Après l'avoir ignoré, les organisations internationales et les pouvoirs publics en redécouvrent les atouts, y voyant une solution miraculeuse à la crise de l'emploi et aux déséquilibres budgétaires croissants.

Au Maroc, le contexte d'aggravation des équilibres budgétaires et financiers de l'État, la montée de la pauvreté et de l'emploi indépendant, la hausse du chômage urbain au cours des deux dernières décennies réactualisent le débat et font une large place à la lutte contre l'informel. Le rôle que ce secteur joue ou qu'on voudrait lui faire jouer est manifeste au-delà des difficultés, non moins manifestes, pour l'appréhender.

Notre recherche consiste à présenter un état des lieux de la microfinance, mais également identifier son origine, évolution et fondement, ainsi que ses effets sur le développement financier du secteur informel au Maroc. D'où la formulation de notre problématique en la question principale suivante :

\section{«Dans quelles mesures la microfinance permet-elle un remède à l'incapacité financière du secteur informel au Maroc? »}

À partir de cette problématique centrale, nous déclinons un certain nombre de questionnements auxquels nous tenterons de répondre tout au long de notre article :

$>$ Quelles sont la genèse et l'évolution de la microfinance ?

$>$ Quelles sont les caractéristiques fondamentales du micro crédit?

$>$ Quelles sont les sources de financement du secteur informel au Maroc ?

Afin de mieux cerner notre recherche et de donner une certaine logique à notre étude, nous allons subdiviser notre tâche selon deux points, un premier traitant l'apparition et l'évolution de la microfinance ainsi que son état de lieux au Maroc et puis dans un deuxième, nous allons mettre en 
lumière les caractéristiques du secteur informel en termes de caractéristiques structurelles et modalités de financement.

\section{Cadre conceptuel de la microfinance}

« Le microcrédit c'est aider chaque personne à atteindre son meilleur potentiel. Il n'évoque pas le capital monétaire, mais le capital humain. Le microcrédit constitue avant tout un outil qui libère les rêves des hommes et aide même le plus pauvre d'entre les pauvres à parvenir à la dignité, au respect et à donner un sens à sa vie ${ }^{1}$.

\subsection{Genèse du concept de la microfinance}

C'est Muhammad Yunus, professeur d'Économie à l'Université de Chittagong au Bangladesh, qui fut le premier en 1975 à initier et lancer le projet des microcrédits. Depuis plus de 45 ans, le microcrédit est un outil au service du développement et de la lutte contre la pauvreté. Il est bien adapté à l'encouragement de micro activités commerciales, artisanales et agricoles. Il est une source d'innovation financière, économique et sociale. Cependant, il ne peut prétendre se substituer ni aux politiques publiques de sécurité sociale ni au développement des infrastructures nécessaires dans les domaines de la santé et de l'éducation. Il peut, par contre, renforcer l'efficacité de ces politiques et de ces programmes grâce à son réseau capillaire dans les quartiers les plus défavorisés et les zones rurales les plus reculées. Plus de 205 millions de personnes dans le monde sont actuellement touchées par le microcrédit, parmi lesquelles plus de 137,5 millions étaient considérés comme faisant partie des plus pauvres, lorsqu'elles ont contracté leur premier emprunt. Parmi ces clients $82 \%$ sont des femmes.

L'impact de la microfinance a fait l'objet de nombreuses études professionnelles et universitaires ; celles-ci ont mis en avant l'importance des services financiers dans la réduction de la vulnérabilité des populations à faibles revenus. L'impact sur la réduction de la pauvreté varie selon les régions et les types de produits financiers offerts et des recherches scientifiques sont en cours pour mieux cerner ces phénomènes.

Ce sont ces bénéfices directs qui expliquent le succès du microcrédit (et de la microfinance de façon générale), sa diffusion rapide dans plus de 80 pays en voie de développement et la reconnaissance internationale qui lui a été accordée, avec notamment l'Année internationale du Microcrédit des Nations Unies en 2005 et l'attribution du Prix Nobel de la Paix au Professeur M. Yunus et à la Grameen Bank en 2006.

\footnotetext{
${ }^{1}$ Vers un monde sans pauvreté, Muhammad Yunus (trad. Olivier Ragasol Barbey et Ruth Alimi), éd. Jean Claude Lattès, 1997 (ISBN 978-2-253-12206-7), p. 399
} 
Parler du microcrédit, c'est d'abord s'interroger sur sa définition. Le Microcrédit consiste à fournir des prêts à court terme à des personnes à très faibles revenus, n'ayant pas accès aux services proposés par les institutions financières classiques, pour les aider à lancer leurs activités ou développer leurs affaires. L'une des caractéristiques spécifiques du microcrédit est qu'il offre, avec un crédit de faible montant, un ensemble d'actions d'accompagnement susceptibles de renforcer les chances de succès de la microentreprise et donc de remboursement du crédit.

Le microcrédit est, d'autre part, associé à un projet. Il en est indissociable, car il est consenti dans le but unique de faire vivre ledit projet. Si la réussite est au bout du chemin, le microcrédit ne sera plus nécessaire : le projet aura permis à une activité génératrice de revenus ou une microentreprise de vivre, d'évoluer et de prendre son essor en recourant désormais aux crédits bancaires classiques.

Qu'y a-t-il de fondamental avec le microcrédit par rapport au crédit classique ?² La différence principale est qu'il est orienté sur une cible nouvelle : les pauvres et les exclus exerçant une activité génératrice de revenus ou désirant créer leur propre très petites entreprises (TPE). Il reconnait leurs talents, leurs besoins et leur capacité à rembourser les prêts. En termes de coût, le microcrédit est plus important que ce que laisserait voir un simple calcul mathématique. Atteindre des clients pauvres et à faibles revenus qui n'ont jamais eu recours à des services bancaires formels demande plus de temps aux agents de développement (par opposition aux agents du crédit du système bancaire traditionnel, parce qu'ils sont chargés non seulement de veiller au processus d'octroi des microcrédits, mais aussi d'accompagner la clientèle par des actions de formation notamment) et plus d'interaction avec les clients, ce qui implique des coûts supplémentaires pour la structure de microcrédit. Il est aussi question de surmonter les problèmes d'analphabétisme.

\subsection{Fondement de la microfinance}

Le client type des services de microfinance est une «personne à faibles revenus qui n'a pas accès aux institutions financières formelles faute de remplir les conditions exigées par ces institutions (documents d'identification, garanties, dépôt minimum...) », (CGAP, 2008).

\footnotetext{
${ }^{2}$ Yunus Center : http://www.muhammadyunus.org/
} 


\subsubsection{Les approches de la microfinance}

Figure 1 : Les approches de la microfinance

\section{Approche maximum}

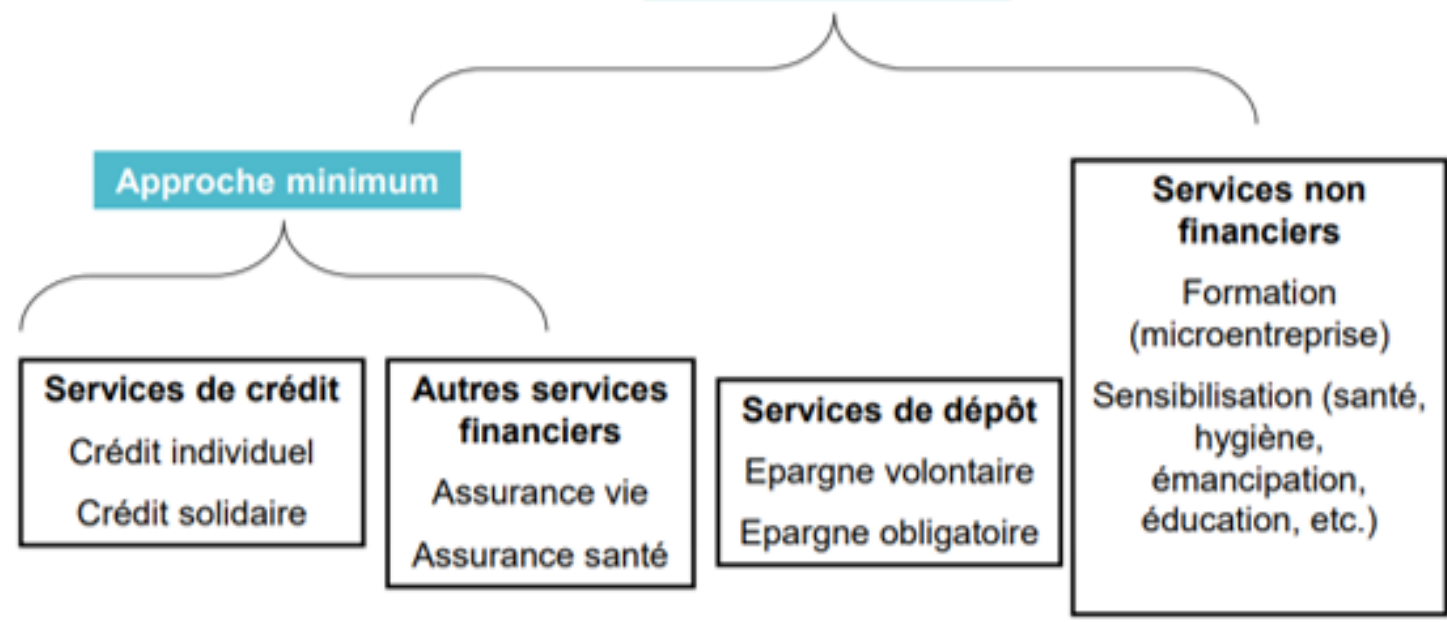

Source : « La finance durable a-t-elle un avenir durable ? $»^{3}$

Le micro crédit occupe une place privilégiée dans l'économie sociale, c'est une activité particulière, car elle est :

$>$ Sociale puisqu'elle participe à l'insertion économique des personnes socialement défavorisées et qui grâce à des petits prêts peuvent créer ou développer leurs propres activités.

Économique, car le micro crédit participe à la promotion de l'emploi et à l'amélioration du tissu économique, les petites unités crées peuvent se développer et s’insérer progressivement dans le secteur organisé.

> Financière, car elle fait appel à des méthodes et procédures de gestion des prêts et ressources très rigoureuses avec comme souci constant, la solvabilité des clients et la rentabilité des fonds à gérer.

\subsubsection{Les enjeux de la microfinance}

Pour le banquier des pauvres; Mohamed Yunus, la crise financière est l'occasion de redessiner le système : «le microcrédit devrait faire partie des droits de l'homme ", et ceci est dû à ses enjeux primordiaux :

Outil efficace de lutte contre la pauvreté très médiatisée qui bénéficie d'une double légitimité au niveau international (Prix Nobel) et local (success stories).

\footnotetext{
${ }^{3}$ www.planetfinance.org
} 
$>$ Des services de qualité et diversifiés (microcrédit, épargne, micro assurance) répondant aux réels besoins des micros entrepreneurs et des populations exclues des systèmes financiers traditionnels.

> Une cible réellement atteinte : femmes, personnes à faible revenu, jeunes, petits producteurs...

$>$ Effet de levier de l'inclusion financière permettant souvent une inclusion plus globale au niveau économique et social.

$>$ Émancipation et indépendance des populations, et rupture progressive des liens de dépendance vis-à-vis de l’État, des fournisseurs, des usuriers...

\subsubsection{Les offres de la microfinance}

De plus en plus les IMF proposent des produits financiers diversifiés afin de répondre au mieux à la demande, mais à cause de leur statut institutionnel, les IMF marocaines n'ont pas encore le droit de collecter de l'épargne. Parmi ces produits on retrouve :

\section{$>$ Les prêts :}

Les crédits pour lancement/développement d'activité sont les crédits les plus octroyés par les IMF. Les micros prêts se divisent en 2 catégories : les prêts individuels, et les prêts solidaires.

Les prêts solidaires sont des prêts accordés à 3-5 entrepreneurs à revenus limités qui forment des groupes selon leurs affinités et qui se cautionnent mutuellement à rembourser leur prêt (qui peut aller de 3 mois à 3 ans, voire plus pour certaines IMF). Chacun des membres du groupe reçoit un prêt individuel dont le montant prêté varie entre 1.000 et 10.000 DH. Les montants sont faibles certes, mais nul ne voudrait se porter garant d'une somme qu'il ne pourra jamais rembourser. Cette technique garantit un taux très élevé de remboursement, puisque chaque emprunteur fait face au jugement de ses collègues et au bouche-à-oreille s'il ne rembourse pas son prêt. Ainsi tous les mauvais emprunteurs sont éliminés. D'autre part, cette technique réduit considérablement les coûts de transaction de l'IMF puisqu'elle prend en charge plusieurs clients en même temps, et comme le remboursement se fait à chaque réunion de groupe, cette technique accroît l'efficacité de l'agent de microcrédit.

Le montant du crédit solidaire reste plus faible que celui qui est proposé dans le cadre des prêts individuels (10000 à 25000 DH). Ce n'est qu'après avoir complété un certain nombre de cycles de prêts solidaires que la personne peut demander un prêt individuel.

Certaines IMF proposent des prêts ciblés pour quelques professions particulières :

- Le Crédit touristique rural : Comme son nom l'indique, ce crédit qui peut aller jusqu'à environ $7.000 \mathrm{DH}$ est destiné aux groupes de micro-entrepreneurs qui ont des activités génératrices de revenus liées au secteur du tourisme, et qui se cautionnent mutuellement.

- Le Crédit d'élevage : Ce type de crédit est caractéristique de certaines IMF comme la fondation ARDI qui a pour but de développer et d'améliorer le rendement de l'agriculture. 
- Le Crédit artisanat : Crédit solidaire à court terme (4 à 8 mois) réservé à des micros entrepreneurs qui pratiquent l'artisanat.

\section{Les Crédits Logements :}

Il a pour but l'aménagement de l'habitation de la personne ainsi que l'amélioration de l'accès à l'électricité et à l'eau. Les montants accordés peuvent aller théoriquement jusqu'à $50.000 \mathrm{DH}$, mais dans le fait, ils dépassent rarement 25.000 DH. Plus concrètement, les clients souhaitant bénéficier de ce prêt ramènent à l'agent de microcrédit des devis de matériaux de construction. Si le crédit est refusé, ils peuvent réclamer une explication.

De manière générale, qu'il s'agisse de prêts ou de crédits logements, les échéances de remboursement sont mensuelles, et s'étalent sur des durées de 6,12, 15, 18 ou 24 mois. Pour certains cas particuliers, la durée de remboursement peut s'étaler sur 7 ans.

\section{La micro assurance :}

Il s'agit d'un système de couverture médicale à 3 paliers (assurance santé basique, complémentaire, et complète) en partenariat avec la Mutuelle Centrale marocaine des Assurances (MCMA). Le client cotise selon le palier auquel il fait partie et la MCMA s'engage à rembourser un pourcentage ou la totalité du prêt en cas de maladie, d'invalidité ou de décès du client. Ces produits restent cependant très peu développés, et de véritables efforts de commercialisation et de vulgarisation doivent être faits auprès des clients traditionnels des IMF pour les sensibiliser à l'importance d'une couverture médicale.

\section{$>$ Les prestations non financières :}

Les différentes IMF proposent aussi des prestations non financières pour soutenir l'activité de leurs clients. La majorité propose un accompagnement personnel et un encadrement aux entrepreneurs, et communique sur la microfinance à leurs clients en publiant des journaux fréquemment. Certaines IMF organisent des formations et des manifestations de sensibilisation en collaboration avec des ONG.

\section{3. État des lieux de la microfinance au Maroc}

Le contexte économique marocain n'est pas très différent de ceux observés dans les autres pays en voie de développement qui ont intégré le système du microcrédit. En fait, l'exclusion sociale et la pauvreté, ainsi que l'existence d'un secteur informel important, constituent, en quelque sorte, les principaux débouchés du secteur marocain du microcrédit.

\section{$>$ Histoire de la microfinance au Maroc}

L'acte de naissance de la microfinance au Maroc a été donné en 1993 par l'Association marocaine de Solidarité et de Développement (AMSED), qui a octroyé le premier microprêt à une femme. Largement inspirées du modèle de MUHAMMAD YUNUS, des associations spécialisées en microfinance sont créées. Des acteurs de la société civile s'engagent sur ce chemin. Noureddine Ayouch 
créera par exemple l'association Zakoura en 1995. Al Karama suivra en 1996, Al Amana et la Fondep verront le jour en 1998. Le programme MICROSTART du PNUD s'implique à partir de 1998 dans le développement de ces associations, et leur offre de l'assistance technique et financière. L'USAID s'implique également, et octroie une aide de 16 millions USD aux organisations non gouvernementales, dont profitera essentiellement Al Amana. La loi de 1999 régissant la microfinance, impose aux ONG généralistes d'isoler leurs activités de microfinance, afin de mieux maitriser les risques, et d'assurer leur viabilité financière. En 2000, l'État réalise l'impact de l'activité des associations de microfinance sur la réduction de la pauvreté, et décide, via le fonds Hassan II, de subventionner le secteur à hauteur de 100 Millions de DH (10 millions $€$ ), permettant ainsi aux associations d'accroître le nombre de bénéficiaires et des montants des prêts accordés.

\section{Le cadre réglementaire : La loi 18-97}

La loi 18-97, entrée en vigueur en 1999, constitue le cadre de référence légal des associations de microfinance au Maroc. Elle définit le microcrédit comme "tout crédit dont l'objet est de permettre à des personnes économiquement faibles de créer ou de développer leur propre activité de production ou de service en vue d'assurer leur insertion économique". Cette loi donne au Ministère de l'Économie et des Finances, le pouvoir de délivrer des licences aux associations de microcrédit, pour octroyer des prêts, mais pas pour collecter l'épargne publique. Elle impose également une viabilité financière des associations de microfinance au bout de 5 ans d'existence, sous peine de retrait de licence et de dissolution. Treize AMF disposent actuellement de cette licence, et aucun cas de retrait de licence ne s'est produit. La loi fixe comme un plafond de prêt établi à $50.000 \mathrm{DH}$ (4500€) à des particuliers, et fixe un taux d'intérêt plafonné, défini annuellement par le Ministère des Finances. Cette dernière clause n'a jamais été appliquée. En 2004, un amendement à la loi 18-97 a permis aux AMF de délivrer des crédits destinés à améliorer les conditions d'habitat des bénéficiaires. La loi prévoit également la création de la Fédération Nationale des Associations du Microcrédit (FNAM), organisme chargé de représenter les associations de microcrédits auprès des instances publiques, d'établir un code déontologique, et de proposer toute action de nature à favoriser le développement de la microfinance. Un conseil consultatif du microcrédit a également été fondé suite à cette loi. Il regroupe des représentants du Ministère des Finances, de la Banque Centrale, de la FNAM, et des banques marocaines. Il est en charge d'examiner les autorisations d'exercice, le montant maximum des microcrédits accordés, le taux d'intérêt maximal pratiqué, ainsi que les ratios de solvabilité des institutions de microfinance. Les acteurs de la microfinance au Maroc s'accordent à dire que le cadre légal de l'exercice de celle-ci a été précurseur et a permis le développement rapide de l'activité, bien que quelques aménagements soient à prévoir, notamment en ce qui concerne la réforme institutionnelle des institutions de microfinance. 


\section{$>$ Analyse financière des institutions de microfinance au Maroc}

L'analyse financière des institutions de microfinance est une tâche particulière. Ce ne sont pas des entreprises traditionnelles qui obligent les actionnaires à profiter, car la loi marocaine les oblige à réinjecter des bénéfices dans leurs fonds propres. Au contraire, les IMF visent à élargir le nombre de leurs bénéficiaires et à améliorer leur suivi, tout en s'assurant d'obtenir de très bons taux de remboursement de leurs clients et d'appliquer les taux d'intérêt les plus raisonnables.

\section{$>$ Les acteurs de la microfinance au Maroc}

Historiquement, les premiers acteurs en microfinance dans le Monde n'avaient pas d'exigences de rentabilité financière. Ceux-ci étaient principalement des autorités gouvernementales nationales, des organisations internationales (PNUD, Union européenne...), des ONG, des agences de développement international (USAID, Agence française de développement...) ... Ce n'est qu'après avoir constaté la viabilité financière, et l'atteinte de niveaux de rentabilité satisfaisants, que des acteurs de la finance classique (Banques, sociétés de financement...) se sont intéressés à la microfinance, soit en finançant directement des institutions de microfinance, soit en créant leurs propres associations de microfinance (Fondation Banque populaire pour le Microcrédit par le Groupe Banque populaire, et Ardi par le Crédit Agricole du Maroc...).

\section{Les bénéficiaires de la microfinance}

Le bénéficiaire de la microfinance est le « Bottom of the Pyramid» (BOP), comme le disait C.K Prahalad dans son livre «The Fortune and the Bottom of the Pyramid». Le BOP peut être un grand nombre de pauvres marginalisés par le secteur capitaliste traditionnel, en particulier le secteur bancaire. En effet, les clients des institutions de microfinance qui ne peuvent pas répondre aux exigences du système bancaire traditionnel par manque de ressources et par méconnaissance sont, en dernière analyse, de véritables entrepreneurs. Ils se tournent vers les institutions de microfinance pour pouvoir investir dans une activité rentable afin de s'assurer d'avoir un revenu financier fixe et de « sortir » de la pauvreté à moyen terme.

En milieu rural, ce sont généralement les agriculteurs et les agriculteurs/éleveurs qui utilisent leurs crédits pour de petits investissements, comme l'achat de vaches laitières, l'obtention de foin... Par contre, en milieu urbain, il en existe plusieurs types. Micro entrepreneurs : commerçants, artisans, vendeurs ambulants, prestataires de services, notamment les femmes entrepreneurs qui mènent des activités pour subvenir aux besoins de leur famille.

\section{Secteur informel et microfinance au Maroc}

Le concept de "secteur informel" est issu du célèbre bulletin d'information de JK Hart, proposé lors de la Conférence africaine sur le chômage urbain (Université du Sussex Research Institute 
Development Research) en septembre 1971, puis révisé et publié par le Modern Journal of African Studies. En 1973. Ce terme a ensuite été adopté par le tout aussi célèbre "Rapport Kenya-1972 Bureau international du travail"

\subsection{Généralité sur le secteur informel}

Nul ne peut nier la place importante qu'occupe l'informel dans le contexte économique des pays. En effet, le marché informel enregistre des performances quantitatives considérables et offres de l'emploi à bon nombre de personnes, ce qui nuit au secteur formel légal et cause de grosses pertes pour les unités productives.

\subsubsection{Cadre conceptuel du secteur informel}

Initialement, dans les années 1950, les tentatives pour comprendre le chômage urbain et les opportunités de revenus en dehors du secteur formel ont conduit à l'identification d'un secteur qui englobait toutes ces activités de subsistance. Le secteur est perçu comme une transition et une simple étape vers l'évolution de l'emploi dans le secteur formel et les zones urbaines. Entre 1960 et 1970, ce secteur est assimilé au «chômage déguisé » ${ }^{4}$. En fait, il est composé de toutes les petites entreprises, artisan, petites familles ou fermes membres pour répondre aux besoins de survie ensemble, et se caractérise par une certaine forme de travail parasitaire, pseudo-salarié, désordre urbain et activités diverses.

La terminologie fut ensuite reprise par le non moins fameux « Rapport Kenya- du Bureau international du Travail » en 1972 qui proposait, à partir des 7 caractéristiques :

- Absence de barrière à l'entrée

- Utilisation des ressources locales

- Propriété familiale de l'entreprise

- Échelle d'activité réduite

- Marchés compétitifs et sans réglementation

- Qualifications acquises hors du système de formation officiel

- Techniques qui privilèges le recours à la main d'ouvres

Ces caractéristiques devaient permettre de distinguer le secteur informel du secteur formel.

Dès qu'on désire choisir une terminologie plutôt qu'une autre, il y a des précisions sur lesquelles l'on devrait s'attarder. L'économie informelle comporte quatre composantes : la production du secteur informel, l'économie souterraine, la production illégale et la production pour usage final propre (Charmes, 2003). Les activités du secteur informel ne s'exercent pas toujours pour fuir les obligations légales.

\footnotetext{
${ }^{4}$ Forme de chômage qui existe du fait que des travailleurs occupent des emplois complètement ou partiellement inutiles, improductifs.
} 
L'économie souterraine renvoie aux activités qui permettent de se soustraire à la réglementation étatique (paiement des impôts, revenus, charges sociales, salaire minimum, les normes d'hygiène et de sécurité).

L'économie illégale renvoie à des activités qui sont interdites par la loi (drogue, prostitution...), soit parce qu'elles sont exercées par des personnes non autorisées (exercice illégal de la médecine), soit encore des activités telles que la contrebande, la contrefaçon, la corruption ou le recel de biens volés. L'interdiction n'empêche pas ces activités d'être exercées au grand jour.

La production pour usage final propre est une composante non marchande de la production de biens par les ménages.

Nous constatons donc que le secteur informel est juste une dimension de ce qu'on appelle «économie informelle ». En traitant du secteur informel, nous soustrayons toutes les activités illégales et ne rentrant pas dans le cadre normal.

\subsection{2. État des lieux du secteur informel au Maroc}

Le Maroc, à l'instar des pays en voie de développement, a connu l'émergence de l'économie informelle à partir des années 1980. Les facteurs derrière cette apparition sont nombreux : évolution démographique forte, exode rural massif, adoption du Programme d'Ajustement structurel (désormais PAS) édictée par les organisations monétaires internationales, incapacité du secteur « moderne » à apporter des réponses et solutions aux besoins sociaux que le secteur informel installait dans le contexte socio-économique national... Tous les facteurs précités ont donné toutes les chances au secteur informel de creuser les chemins et de s'accroître. Ce dernier finira de devenir une composante dynamique et durable de l'économie marocaine. Pour certains observateurs, le secteur informel a la vie dure au Maroc, ils estiment qu'il représente, officiellement, près de $25 \%$ de l'économie nationale. D'autres études et enquêtes parlent de 30 à 35\%. Le nombre d'unités abritées par le secteur est estimé à environ 1,55 million d'unités, avec un nombre d'emplois de près de 2,5 millions de mains d'œuvre. Chaque année 40.000 unités viennent s'ajouter pour opérer dans le secteur.

Figure 2 : Évolution de l'indicateur composite de l'informalité et du proxy de la population informelle de 2007 à 2020

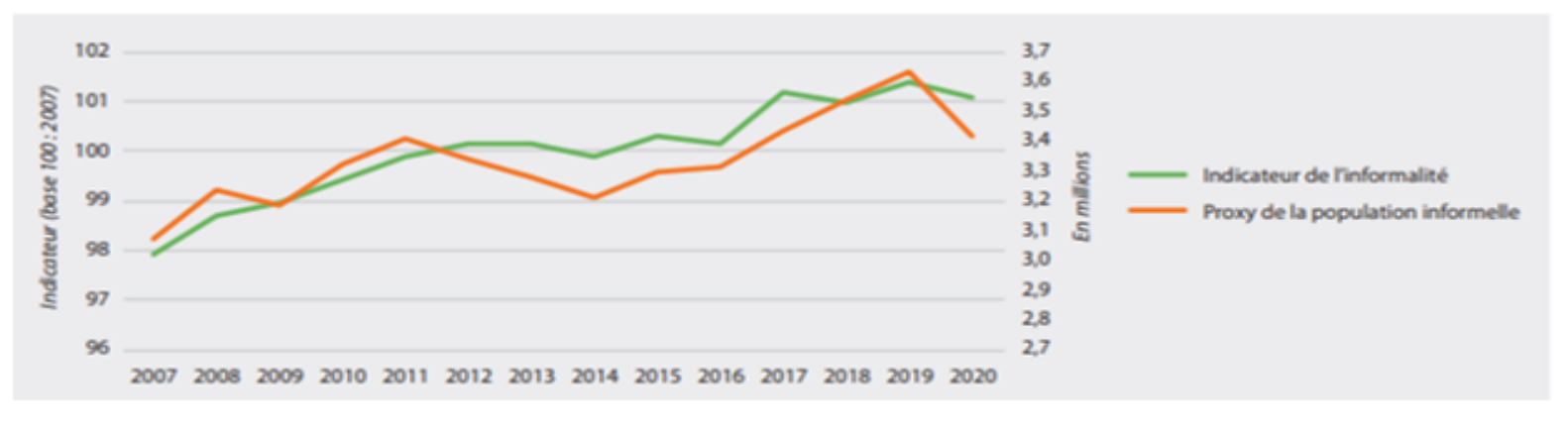

Source : « Haut-commissariat au plan $»^{5}$

\footnotetext{
5 «Le secteur informel au Maroc : principales caractéristiques et tendance d'évolution », Lamiaa EL RHAZ, Yasmine BOUZINEB, Division des études générales, DPP-HCP
} 
L'indicateur composite de l'informalité est influencé positivement par le développement de l'autoemploi et par une augmentation de la TVA et du SMIG. En revanche, une amélioration de la croissance du RNB par habitant et de la qualité de la réglementation permet de réduire l'informalité. Les évolutions de l'indicateur composite de l'informalité et du proxy de la population informelle sont synchrones sur plusieurs points. En effet, elles sont pratiquement parallèles et affichent une similitude quant aux périodes de retournement.

En 2020, les deux indicateurs ont connu une baisse notable, dans le sillage des restrictions mises en vigueur pendant la période de confinement et de la faible reprise de la demande des ménages en période post-confinement. Dans une conjoncture différente de celle de la crise sanitaire, cette baisse de la population informelle aurait été synonyme d'une stratégie efficace de renforcement de l'attraction du secteur organisé et d'une transition soutenue vers la « formalité ». Cependant, compte tenu de la nature de la crise, cette baisse ne peut traduire qu'un basculement des actifs de l'informel dans le chômage ou l'inactivité.

La crise sanitaire actuelle a mis en exergue les vulnérabilités du secteur informel et l'importance des aides publiques à déployer pour soutenir le pouvoir d'achat de ses effectifs employés. Ainsi, bien qu'il offre une solution alternative au chômage et au manque de revenu, sa structure de fonctionnement et sa productivité ne permettent pas son développement économique et n'assurent pas une protection sociale face aux crises économiques. Pour renforcer la résilience de ce secteur et améliorer sa performance économique, une stratégie nationale dédiée aux activités informelles reposant sur une approche intégrée et cohérente est indispensable. Elle doit tenir compte pour cela de plusieurs dimensions dont notamment l'accès au financement et au marché, la formation, la sécurité sociale, la réforme du dispositif fiscal, notamment celui qui concerne le mécanisme de la TVA, et l'amélioration de la gouvernance, surtout en matière d'allègement et d'adaptation de la réglementation en vigueur.

\subsection{Structures financières des unités de production informelles au Maroc}

Il est de tradition, selon une optique dualiste, de considérer les pays en développement comme caractérisés par l'existence deux secteurs financiers, l'un, formel, qui répond aux besoins du secteur moderne monétisé et l'autre, informel. Le premier, organisé et axé sur les zones urbaines répondrait aux besoins financiers du secteur moderne monétisé, cependant que le second, inorganisé, régirait les sphères de subsistance. Au Maroc, les institutions financières formelles n'interviennent quasiment pas dans le financement du secteur informel, que ce soit lors de la phase de démarrage ou pour accroître ses investissements. Le Maroc est d'ailleurs loin d'être un cas isolé, et nombre de travaux portant sur l'Afrique et l'Asie ont relevé des observations similaires (Lellart, 2003). Les banques ne s'intéressent pas à ces formes d'activité, et l'essentiel du financement s'effectue hors banque, en recourant aux circuits non institutionnalisés. 


\subsubsection{Financement lors de la création}

Les pratiques de financement dans le secteur informel relèvent essentiellement d'un phénomène local et endogène. Le démarrage de l'activité s'appuie sur des réseaux sociaux particulièrement mis à contribution. Ces pratiques semblent particulièrement adaptées aux normes du milieu et aux réalités locales. Les circuits de financement hors banque sont marqués par leur hétérogénéité. Parmi leurs caractéristiques figurent la prédominance des transactions en espèces, l'échelle limitée des opérations, l'absence de réglementation et leur adaptabilité. Les différentes origines de la mobilisation des fonds, lors de la création des microentreprises, apparaissent dans le tableau suivant.

Tableau 1 : Sources de financement de la création de l'unité de production informelle

\begin{tabular}{|l|c|}
\hline \multicolumn{1}{|c|}{ Source de financement } & $\%$ \\
\hline Héritage & 4,7 \\
\hline Epargne personnelle & 56,4 \\
\hline Vente de biens personnels & 2,0 \\
\hline Crédit bancaire & 1,1 \\
\hline Micro-crédit & 2,2 \\
\hline Crédit d'autrui & 19,0 \\
\hline Transfert reçu & 3,6 \\
\hline Départ volontaire & 0,2 \\
\hline Autres & 10,9 \\
\hline
\end{tabular}

Source : Enquête nationale sur le secteur informel, HCP

On peut identifier plusieurs types d'intervenants dans le financement des unités. Il ressort que le financement individuel, hors institution bancaire, sur la base d'une épargne personnelle, constitue la source de financement dominante lors de la création de la microentreprise. Il s'agit soit d'une épargne personnelle réalisée lors de l'exercice d'une activité antérieure à la faveur d'un emploi dans des petits métiers ou en tant que salarié ou encore fruit d'une thésaurisation. La prépondérance de cette forme confirme l'échelle restreinte des activités informelles qui peuvent être financées, pour nombre d'entre elles, sans grandes difficultés, puisqu'elles n'exigent qu'un faible capital de départ (ambulantes, petites activités sous-traitantes...) ou que quelques outils ou machines. Le financement provient aussi de transferts reçus des réseaux familiaux et amicaux ou d'héritages qui sont révélateurs de la résistance du système traditionnel d'entraide. Ces modalités n'offrent, cependant, que des possibilités limitées. En réalité, épargnes personnelle et familiale peuvent être confondues. Elles prennent la forme de dons ou 
de prêts sans intérêt qui n'imposent aucune échéance de remboursement. Ceci se justifie, car la décision de créer une microentreprise est, dans nombre de cas, dictée par l'environnement familial (reprise d'une affaire familiale). Enfin, il faut ajouter que les crédits octroyés par autrui sont non négligeables.

L'apport du microcrédit en tant que source de financement est insignifiant. Il est essentiellement le fait des ONG qui interviennent à travers des projets de lutte contre la pauvreté, pour favoriser le développement de l'activité et l'émergence de petites entreprises. En effet, le Maroc est le pays du Maghreb où le microcrédit est le plus développé et où les institutions de microcrédit sont les plus nombreuses. Les premiers programmes de prêts en direction des personnes à bas revenus destinés à financer des activités relevant essentiellement du secteur informel ont démarré dans les années 1993 1994. Le recours au crédit bancaire reste très faible et plusieurs faits expliquent les faibles relations entre l'informel et le système bancaire. En premier lieu, les institutions bancaires ont un comportement en matière d'affectation et de mobilisation des ressources qui privilégie le secteur public et le secteur privé formel. En second lieu, une attitude négative des micro-entrepreneurs à l'égard du système officiel qui à leurs yeux présente des contraintes, telles les garanties exigées envers des catégories qui sont souvent insolvables et pour qui le système bancaire privilégie les grandes entreprises. En troisième lieu, l'inadaptation des instruments financiers aux besoins des unités informelles, tant au niveau des montants alloués que des garanties exigées. D'autres contraintes pèsent sur les micro-entrepreneurs telles que la lourdeur et le coût des procédures (les frais de constitution d'un dossier et le nombre d'imprimés exigés par la banque rendent les procédures opaques), les échéances de remboursement mensuelles, alors que l'activité est irrégulière ou saisonnière. D'une manière générale, les micro-entrepreneurs ne maitrisent pas les circuits financiers formels.

Une des conséquences du faible accès aux liquidités lors du démarrage est qu'il se crée des différenciations entre catégories au sein du secteur informel lui-même, entre les patrons, d'un côté, et les salariés et apprentis, de l'autre. Si les premiers peuvent compter sur l'épargne constituée antérieurement, en tant que salariés ou avec l'appui familial, les seconds peuvent difficilement surmonter les barrières financières pour s'installer. Dans le même sens, ces barrières provoquent des différenciations entre les patrons et une partie des indépendants, d'un côté, et les indépendants de l'autoemploi qui forment la grande majorité, de l'autre.

\subsubsection{Financement lors du fonctionnement}

Comme lors de l'installation, les institutions officielles ont un rôle insignifiant dans le financement durant le fonctionnement, ou ce que l'on peut appeler investissement lors du cycle de production, qui s'appuient alors sur les circuits non institutionnels et sur une économie de proximité géographique et sociale. On peut identifier les pratiques suivantes : 
$>$ L'autofinancement : Cette pratique est dominante, car l'endettement est mal perçu, quelles que soient l'activité et la taille de celui-ci. Certains entrepreneurs préfèrent « compter sur leurs propres forces » ne pas courir le risque de l'endettement.

$>$ Le crédit personnel fournisseur appelé talk est une pratique très courante dans la quasi-totalité des activités. Le talk semble constituer une alternative au manque de liquidités et à l'absence de recours au crédit bancaire. Il s'agit d'une pratique de financement de type commercial qui trouve son sens dans les relations entre fournisseurs et commerçants : les fournisseurs acceptent de n'être réglés qu'ultérieurement pour avoir plus de chance de vendre à nouveau. De la sorte, les petites entreprises et les prestataires de services se trouvent dans une position où ils sont à la fois créditeurs et débiteurs. Cette pratique est fortement répandue parmi les artisans et les informels qui n'ont pas les moyens de constituer des stocks. Le talk est largement sollicité en raison des avantages qu'il offre aux partenaires : la modicité des montants en jeu, les délais de remboursement qui peuvent aller de trois jours à deux mois, un type de financement adapté au caractère instable des activités et, enfin, un système caractérisé par une grande souplesse, la facilité de l'accès à l'argent sans aucune procédure. Ce système ne serait pas possible sans la force des relations personnelles, les rapports de confiance et de moralité des bénéficiaires dans une économie de proximité.

$>$ Le recours aux associations ponctuelles : En cas d'absence de liquidités, les mécanismes d'adaptation qui se mettent en place peuvent prendre la forme «d'associations » informelles, immédiates, qui se créent pour l'achat d'un stock de marchandises ou de matières premières.

$>$ Le crédit personnel solidarité : Il semble inexistant. En effet, les formules de type tontine ${ }^{6}$ tel qu'elles existent en Afrique n'interviennent pas comme source de financement, vraisemblablement en raison de l'affaiblissement des liens de solidarité. L'auto-organisation de type « Daret» comme alternative pour financer l'activité est inexistante dans le secteur informel. Quand on la retrouve, c'est davantage pour faire face à des événements imprévus comme les enterrements.

> Le «banquier artisan associé » (maalem choukara). C'est une personne extérieure au métier (agriculteur, petit fonctionnaire, commerçant, travailleur à l'étranger) qui peut engager des fonds de diverses manières. Par le financement du local ou du matériel et dans ces cas, la gestion est confiée à l'artisan ou à un gérant. Le banquier artisan peut intervenir en équipant le local et

\footnotetext{
${ }^{6} \mathrm{La}$ tontine constitue un faisceau de créances et de dettes mutuelles qui s'équilibrent constamment et qui disparaissent à la fin du cycle. Les participants qui s'auto-organisent décident ensemble du montant des cotisations, de leur durée, des modalités de la levée, mais aussi des amendes en cas de retard, pour alimenter une caisse de secours ou de prêt (Lellard, 2003) ... Ce sont ainsi les règles mises en place par les membres qui organisent l'accès au financement.
} 
en finançant la main-d'œuvre. Enfin, il peut s'associer temporairement au micro-entrepreneur pour réaliser une opération ponctuelle en lui accordant un crédit en contrepartie d'une participation aux bénéfices, selon un accord préétabli.

> Le recours au crédit bancaire : Il est faible (4,8\%), de même que le microcrédit $(2,2 \%)$. Comme explicité plus haut, il existe une attitude plus ou moins négative à l'égard du système officiel pour les mêmes raisons que celles évoquées lors de l'installation, en particulier dans les activités se survie. Ceci ne veut pas dire que certaines activités n'aient pas de relation avec le système officiel. Ce recours est nécessaire quand il s'agit de recouvrer ou d'encaisser un chèque de garantie, comme ceci est le cas des activités évolutives où on note une interpénétration entre les deux systèmes, formel et informel, de financement. Dans la réalité, si les activités de survie ne perçoivent pas l'intérêt du recours au système bancaire, d'autres activités en revanche le perçoivent, dans une perspective de formalisation et de passage à une échelle supérieure.

Ce que l'on peut retenir, ce sont les pratiques endogènes qui dominent au niveau du financement. Cellesci présentent certains avantages par rapport au crédit officiel. Elles s'appuient sur la proximité, présentent une certaine souplesse et sont adaptées au milieu, mais le revers, c'est que ce circuit est risqué, de même que le faible accès aux circuits bancaires handicape les microentreprises et constitue une barrière à l'entrée.

\section{Conclusion}

Le secteur informel continue à poser un défi, tant au niveau de l'analyse que dans la réalité. La réflexion menée montre que la dynamique des activités du secteur informel ne saurait être appréhendée exclusivement à travers les fonctions économiques qu'il assure, même si celle-ci est vitale. Réduire l'analyse à cette seule dimension ne permet pas de prendre la mesure des enjeux réels, sociaux et institutionnels en particulier.

Sur le plan théorique, les activités du secteur informel ne sauraient trouver leur expression théorique ni dans le cadre unidimensionnel du marché ni dans le cadre de la surexploitation. L'appréhension du secteur informel, en dépit de la faible capacité analytique de cette notion, est rebelle aux cadres d'analyse globaux. La singularité des institutions et la pluralité des normes, des codes et des organisations dans lesquelles évoluent ces activités ont mené à les considérer comme un procès institutionnalisé.

$\mathrm{Au}$ niveau macro-économique et en se situant dans une perspective historique, la dynamique du secteur informel n'est pas sans liens avec le régime d'accumulation et les politiques de développement mise en œuvre. Il en a découlé un processus d'informatisation du marché du travail officiel et une complexification de l'emploi. Les manifestations les plus évidentes en sont le recul de l'emploi officiel, la réallocation des effectifs vers des secteurs soumis à l'informatisation, la régulation du marché du 
travail par la montée des formes non salariales, la baisse du salariat et son caractère instable, la montée du chômage, de nouvelles modalités du travail féminin et une mobilité sectorielle des actifs.

Pour conclure, et en réponse à notre question centrale de départ, on peut avancer et confirmer que l'économie informelle et en dépit, de son caractère nuisible, rebelle, perturbateur...reste un catalyseur de développement des économies sous-développées. Au Maroc, le secteur s'impose comme un secteur de survie pour une grande marge de la population, un défi devant les autorités étatiques, un manque à gagner. La lutte contre ce fléau doit se faire en termes d'intégration moyennant, entre autres, des mesures et incitations diverses et variées (fiscales, comptables, financières, juridiques...) et institutionnelles. Ces dernières années les efforts à intégrer l'économie informelle ne sont pas à nier, mais il reste beaucoup à faire, car le secteur échappant à tous suivis et mesures peut présenter des surprises.

\section{BIBLIOGRAPHIE}

[1] Ashta, Arvind (2016): «Microfinance: Battling a Wicked Problem »

[2] Yunus Center : http://www.muhammadyunus.org/

[3] Ayuk, T. (2015) : «La microfinance en Afrique centrale : Le défi des exclus »

[4] Mutisya, Musau, (2014): «Access to Microfinance and Financial Training for Innovative Urban Sustainability : Collective Investments at the Bottom of the pyramid segment in urban Kenya»

[5] Boyé, Sébastien,Hajdenberg, Jérémy,Poursat, Christine (2009): «Le guide de la microfinance : Microcrédit et épargne pour le développement Ed. 2 »

[6] Clara ATALLAH, Omar EL HYANI, (2009) : «Microfinance : Quelles perspectives de développement pour les IMF : cas du Maroc »

[7] François Roubaud (2014) : «L'économie informelle est-elle un frein au développement et à la croissance économiques? », pages 109 à 121

[8] Khalid ROUGGANI, Youness BOUTHIR (2014) «SECTEUR INFORMEL - ÉCONOMIE INFORMELLE : QUELLE CONTRIBUTION POUR LE DÉVELOPPEMENT DE L’ÉCONOMIE MAROCAINE ? »

[9] Abdelaziz KHALFAOUI, Siham LAMARI : «Les unités de production informelles au Maroc : une analyse comparative à l'échelle régionale » 